\title{
Deterioro de la universidad venezolana en los últimos 15 años
}

\author{
LUIS FUENMAYOR TORO
}

Médico Cirujano, Universidad Central de Venezuela, 1968. Especialista en farmacología (farmacólogo), Universidad Central de Venezuela, 1970, Doctor of Philosophy (PH.D.), Universidad de Cambridge, UK, 1979. Profesor Titular, Universidad Central de Venezuela, 1983. Líneas de investigación: neuroquímica, neurofisiología y conducta motora; políticas públicas en educación universitaria, ciencia y tecnología y salud pública.

\begin{abstract}
Agradezco a los profesores Ernesto Fuenmayor (UCV), Carmen Carrasquel (ULA) y María Luisa Maldonado (UC) por la recopilación de parte de los datos presentados.
\end{abstract}

\section{Resumen}

Se analizan trabajos e investigaciones a los cuales se añaden datos estadísticos de algunas instituciones para ilustrar el grave deterioro actual de la universidad venezolana. La severa reducción de sus cursantes de pregrado y postgrado, la disminución de sus egresados, la escasez de profesores investigadores, el deterioro de la infraestructura, la obsolescencia del equipamiento, el desabastecimiento de las bibliotecas, la bajísima asignación presupuestaria y la inseguridad personal y de las instalaciones caracterizan la situación universitaria actual. Esta ha sido generada por políticas educativas infames desplegadas desde 2005 agravada luego por la intensa crisis económica que atraviesa el país. La recuperación será una tarea de décadas, pues se trata prácticamente de reconstruir desde el principio.

Palabras clave

Universidad; presupuesto universitario; estudiantes; egresados; profesores; sueldos; Venezuela 


\title{
Growing deterioration of venezuelan university over the past 15 years
}

\begin{abstract}
In this work, research papers and statistical data from some other educational institutions are analyzed to illustrate the current deterioration of the Venezuelan University: the severe reduction of its undergraduate and postgraduate students, the decrease of their graduates, the shortage of research professors, the infrastructure deterioration, the equipment obsolescence, the inadequate library supplies, the insufficient budget allocation, and the insecurity of facilities and human resources. This situation has been produced by infamous education policies, implemented since 2005, which was aggravated by the national economic crisis. Recovery will be a decades-long task, as it is practically about a reconstruction from the scratch.
\end{abstract}

Keywords

University; University budget; Students; Graduates; Academic salaries; Venezuela.

\section{Deterioração da universidade venezuelana nos últimos 15 anos}

\section{Sumário}

São analisados trabalhos e pesquisas aos quais são adicionados dados estatísticos de algumas instituições para ilustrar a grave deterioração atual da universidade venezuelana. A severa redução dos seus alunos de pré-graduação e pós-graduação, a diminuição dos seus graduados, a escassez de professores pesquisadores, a deterioração da infraestrutura, a obsolescência do equipamento, o desabastecimento das bibliotecas, a baixíssima alocação orçamental e a insegurança pessoal e das instalações, caracterizam a situação universitária atual. Ela tem sido gerada por políticas educativas infames estendidas desde 2005, e foi agravada logo pela intensa crise econômica que atravessa o país. A recuperação será uma tarefa de décadas, pois trata praticamente de reconstruir desde o início.

\section{Palavras chave}

Universidade; orçamento universitário; estudantes; graduados; professores; salários; Venezuela. 


\section{Introducción}

Uno de los graves problemas en la Venezuela actual, para precisar el estado en que se encuentra el sector universitario venezolano o cualquier otro sector de la sociedad, es la dificultad de los investigadores y sus equipos para deslastrarse de las distorsiones creadas por los prejuicios existentes y las posiciones asumidas en la lucha política del presente. La beligerancia política ha alcanzado niveles alarmantes dada la polarización vigente en el país desde hace unos 20 años, que obstaculiza una visión clara y objetiva de la realidad.

Así, se llega a negar verdades más que evidentes en el afán de descalificar al contrario. En el sector universitario, por ejemplo, el gobierno y sus seguidores afirman que la universidad venezolana ha mantenido excluidos a los sectores populares, lo cual no es respaldado en absoluto por las investigaciones del último medio siglo. Por su parte, para la oposición extremista la creación de un sistema universitario paralelo al existentes en 1998 es una aberración nunca vista en el país, lo cual también es falso pues, a partir de 1970 se crearon las llamadas universidades experimentales como un sistema paralelo al de las universidades autónomas existentes.

Otra dificultad para el estudio del sector, esta vez instrumental, es la ausencia de publicaciones oficiales con la información estadística del mismo. Para 2004, existían muchas publicaciones periódicas con los datos estadísticos actualizados del sector, oficial y privado, gracias al esfuerzo sostenido desde 1999 por la Oficina de Planificación del Sector Universitario (OPSU) y el Secretariado Permanente del Consejo Nacional de Universidades (CNU) (Tabla 1). Al ser descontinuadas estas publicaciones, el sector educativo superior ha quedado sin la información necesaria para su evaluación permanente.

A pesar de estas limitaciones presentamos el panorama actual de la universidad venezolana, principalmente en el área académica y en la situación de la comunidad universitaria, es decir, de sus profesores y estudiantes, el financiamiento y la planta física. Ilustraremos con algunas cifras no publicadas y datos concretos el enorme deterioro ocurrido y su desarrollo temporal, el cual es tan obvio que más que una demostración requiere de una valoración de su gravedad. Analizaremos la información de investigaciones científicas, así como las publicadas por las instituciones y el gobierno y las obtenidas de materiales informativos institucionales y de datos gentilmente aportados por el personal académico y técnico de las instituciones.

\section{Breve reseña histórica}

En 1958 había en Venezuela tres universidades oficiales, dos de ellas coloniales y dos privadas de muy reciente creación. La matrícula total era de unos 12 mil estudiantes y las carreras profesionales estaban restringidas a las más tradicionales, con postgrados limitados al área de la salud pública y sin mayor investigación científica (Fuenmayor Toro, 2002).

Para 1998, el número de universidades oficiales era de $18(+600 \%)$, las privadas de $20(+1000 \%)$, la matrícula estudiantil de unos 600 mil cursantes $(+5,000 \%)$, sin contar los 280 mil estudiantes de carreras cortas de los ins- 
titutos tecnológicos y colegios universitarios, antes inexistentes. El postgrado se desarrolló y su número alcanzó el millar de programas en el sector oficial y unos 250 en el privado. Este aumento fue a expensas de las especializaciones y luego de las maestrías, en tanto el doctorado quedó rezagado (Fuenmayor Toro, 2002).

En este mismo lapso se crearon las carreras de tres años de duración dictadas en institutos (31) y colegios universitarios (8) oficiales y sus equivalentes privados (55), conducentes al grado de técnico superior universitario. La tasa de atención a la población, entre 20 y 24 años de edad, creció desde alrededor de un 3\% para finales de los años cincuenta, hasta 33\% en 1995 (OPSU, 2001, p. 5). Se instrumentó la carrera académica con un escalafón de cinco niveles, concurso de oposición público para el ingreso y defensa pública ante un jurado de un trabajo de investigación original para el ascenso. Los sueldos variaban entre 800 dólares para el primer nivel y 3,000 para el último. El total de docentes se elevó a unos 40 mil, de los que 8 mil eran del sector privado (Fuenmayor Toro, 2002).

Este crecimiento requirió de un importante aumento en la asignación presupuestaria, la cual creció 25 veces entre 1964 y 1981, al pasar de 43.29 millones de dólares a 1,050.47 millones, lo cual no solo no pudo sostenerse posteriormente, sino que sobrevino una limitación financiera importante a raíz de la grave crisis económica ocurrida en Venezuela a partir de 1983 (Fuenmayor Toro, 2002).

Toda esta descripción debería obviar cualquier discusión sobre si hubo o no un desarrollo positivo de la educación universitaria venezolana en el período 1958-1998, pese a la contracción habida en las últimas dos décadas del siglo XX.

\section{La universidad venezolana en el siglo XXI}

Período 1999-2004: García-Guadilla, Montilva, Acedo, Lepage y Otero (2006) describen las políticas y las acciones oficiales en el sector universitario nacional a partir de 1999, cuando asume la presidencia de Venezuela el teniente coronel Hugo Chávez Frías. Para estos autores hubo un primer período, 19992004, en el cual las políticas públicas hacia la educación universitaria y la dirección de los organismos oficiales encargados de la misma estuvieron a cargo de académicos, procedentes en su mayoría de la Uni- 
versidad Central de Venezuela (UCV), que habían ejercido funciones académico-administrativas y gremiales y que, por lo tanto, conocían muy bien el sector educativo superior. Se trataba de profesores investigadores que "en la década de los ochenta y los noventa lideraron la política universitaria desde sus propias instituciones académicas con posiciones de rechazo hacia la política oficial de entonces, calificada de neoliberal, y proponiendo alternativas" (García-Guadilla et al., 2006, p. 8).

Las políticas y sus ejecutorias se instrumentaron a través de varios programas y proyectos, principalmente de la opsu, entre los que destacó el Proyecto Alma Mater para el mejoramiento de la calidad y la equidad de la educación universitaria en Venezuela (OPSU, 2001) y estuvieron dirigidos a:

- Elevación de la calidad académica mediante programas en todas las universidades oficiales. Creación del sistema de evaluación y acreditación, fortalecimiento de la carrera académica profesoral, ejecución de un programa de formación de doctores, soporte financiero y equipamiento de las unidades de investigación existentes, dotación de las bibliotecas principales de escuelas y facultades de todas las universidades con los libros de texto de los estudiantes y dotación de salas de computación con acceso a internet para uso estudiantil como sitios de trabajo y búsqueda y no como laboratorios de enseñanza (OPSU, 2001; García-Guadilla et al., 2006).

- Garantizar la equidad en el ingreso estudiantil, perfeccionando el instrumento de selección existente (Prueba de Aptitud Académica), uso de los promedios de notas normalizados por planteles para evaluar el rendimiento de los aspirantes, diseño e instrumentación de una prueba de exploración vocacional, otorgamiento de becas a los seleccionados de los niveles socio-económicos "D” y "E” y seguimiento institucional de su rendimiento. Además, se construyó la Aldea Universitaria de Santa Elena de Uairén (Fuenmayor Toro, 2004), programa a ser replicado en municipios fronterizos para dar educación de calidad a poblaciones históricamente marginadas; proyecto que fue más tarde distorsionado y envilecido en su aplicación en el resto del país (OPSU, 2004; García-Guadilla et al., 2006).

- Financiamiento oficial de las universidades mediante un modelo de asignación presupuestaria calculado sobre bases técnicas con indicadores y fórmulas, que fue discontinuado a partir de 2005 (García-Guadilla et al., 2006).

- Construcción de nueva planta física, conclusión de obras paralizadas por años, reparación y remodelación de edificios, laboratorios y aulas de docencia e investigación en todas las universidades autónomas y experimentales (García-Guadilla et al., 2006). Se presenta a manera de demostración los resultados de este programa en la Universidad de Carabobo, en trabajo con sus rectores para el lapso 2000-2008 (Tabla 2). Esta institución hizo excelente uso de los recursos financieros asignados para este fin. Al igual que ella, todas las universidades se beneficiaron de este programa. 
- Regularización institucional. Legalización o cierre de sedes y programas no autorizados en universidades oficiales y privadas; mejoramiento del transporte estudiantil universitario con la dotación de unidades de transporte colectivo, creación de la Universidad Bolivariana de Venezuela, puesta en marcha de la Universidad de Yaracuy, transformación en universidades de tres instituciones académicas existentes que reunían los requisitos a tal efecto (García-Guadilla et al., 2006) y creación de cuatro institutos universitarios de tecnología.

"Todos los programas mencionados anteriormente fueron descontinuados a finales de 2004, cuando el Director de la OPSU y todo su equipo fueron desplazados" (García-Guadilla et al., 2006, p. 10) al ser designado ministro un funcionario sin experiencia en el sector, sin conocimiento del mismo y con una línea de asumir la educación universitaria con criterio politiquero, ideológico y clientelar. Se abandonó la calidad académica y se indujo una masificación desordenada sin atender los requerimientos de personal académico formado en número suficiente, ni las exigencias de aulas, laboratorios, equipamiento y bibliotecas. Otro tanto ocurrió con los programas, menos numerosos y extensos, del Viceministerio de Políticas Académicas, los cuales también desaparecieron al ser sustituido el equipo dirigente del mismo (García-Guadilla et al., 2006).

Período 2005 en adelante. Hasta mediados de 2003, el presidente Chávez no se había ocupado directamente de la educación universitaria, lo que permitió realizar los programas académicos señalados. Para 2003, el jefe de Estado ya había logrado disipar las conspiraciones que la oposición había desarrollado desde 2001, entre ellas, la huelga general de ese mismo año, el golpe de Estado de abril de 2002, el paro general y sabotaje petrolero de finales de 2002 y principios de 2003. Al salir victorioso de estos incidentes, Hugo Chávez controla totalmente la Fuerza Armada Nacional, fundamental en la estabilidad política del país, y Petróleos de Venezuela (PDVSA), de cuya actividad vive económicamente la nación venezolana. Dispone entonces de tiempo para comenzar a ocuparse de otros sectores, el educativo universitario entre ellos.

Es el año de la creación de la Universidad Bolivariana de Venezuela, la universidad insigne del chavismo, que luego de un corto extravío académico inicial, asume una función claramente ideologizada y partidaria dirigida a sus estudiantes, profesores y trabajadores. Baste decir que el rector que sustituye a la rectora fundadora fue un licenciado en educación con pocos años de graduado, sin experiencia ni carrera académica y designado a medio tiempo, pues el resto de su dedicación lo cumplía como viceministro. Es el año también de la creación de la Misión Sucre, supuestamente pensada para atender y nivelar a los estudiantes de bajos estratos excluidos del sistema, pero realmente dirigida a la creación de un programa de ingreso paralelo, donde no se tenía que cumplir con los requisitos establecidos para el resto de los aspirantes (García-Guadilla et al., 2006) [...] 


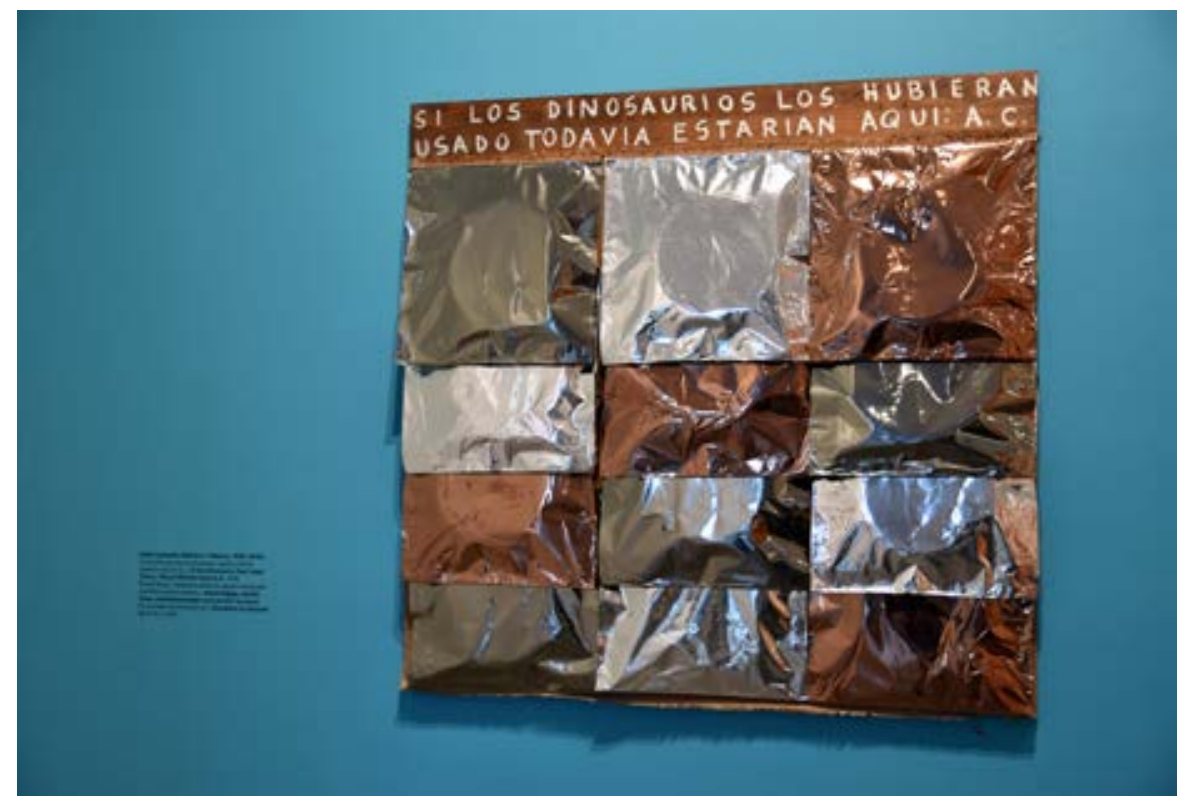

Dentro de la Misión Sucre aparecen las llamadas aldeas universitarias, distorsión perversa de la idea instrumentada exitosamente en la Gran Sabana, estado Bolívar, pero con el propósito de construir todo un para sistema paralelo universitario sin control del $\mathrm{CNU}$, sin posibilidades de evaluación, sin rendiciones de cuenta, sin datos estadísticos, sin resultados importantes (García-Guadilla et al., 2006) y donde las inversiones mil millonarias se administrarán sin control. Secuestran recursos del sector formal y los desaparecen con total impunidad. Otro programa ficticio fue el de los Fundos Zamoranos Universitarios, hecho como fachada de una educación universitaria socio productiva que respondía a las comunidades menos favorecidas. De este programa no se conocen su evaluación, ni resultados, ni existe evidencia del trabajo hecho (García-Guadilla et al., 2006).

A partir de 2005, el gobierno se embarcó en la creación compulsiva de universidades, para lo cual transformó los colegios universitarios y los institutos de tecnología en universidades territoriales sin importar si reunían las condiciones para ello. Al mismo tiempo desplegó un hostigamiento de las universidades autónomas y de aquellas experimentales que no controlaba, las que fueron sometidas a severas limitaciones presupuestarias, a la paralización judicial de sus procesos electorales, a la asignación de sueldos miserables a sus docentes y trabajadores, al deterioro de su planta física y de los servicios y a las agresiones vandálicas de grupos parapoliciales violentos.

Claramente el gobierno nacional torció radicalmente el rumbo de la gestión educativa superior que llevó hasta 2004, inclusive. Con la excusa de combatir la universidad burguesa, el Ministerio de Educación Superior relegó, el conocimiento científico y la academia para privilegiar la demagogia, la violación de leyes y normas y la corrupción (Fuenmayor Toro, 2009). 
Los problemas universitarios se agravaron y las instituciones casi cierran el total de sus actividades.

A estas acciones perversas se unieron las aberraciones ejecutadas por funcionarios de distintos niveles. Del lado universitario nos encontramos con la desidia de autoridades y la débil y escasa resistencia de la comunidad ante este estado de cosas, que se explica por la fuerte represión desatada por los cuerpos de seguridad del Estado, la judicialización de las protestas y las agresiones delictivas de grupos parapoliciales (Fuenmayor Toro, 2009). El grave deterioro vigente no es entonces fortuito, sino de esperarse y muy anterior a la aparición de la crisis financiera actual.

\section{Equidad sin calidad: un engaño recurrente}

En su ideologización paranoica, el gobierno trata el problema de la equidad en el ingreso sin atender a todos los cambios positivos que se habían efectuado en los primeros años de su propia gestión gubernamental. Se abandona el sistema que había sido diseñado y establecido y que garantizaba equidad con calidad en el acceso estudiantil a las universidades (Fuenmayor Toro y Mejías, 2004; Sarco-Lira y Fuenmayor Toro, 2004). Se adopta, a partir de 2008, un sistema que llevó a las condiciones socioeconómicas a determinar el $50 \%$ del puntaje necesario para ser seleccionado (Parra-Sandoval y Núñez-Torres, 2016; Luis, Cortázar y Fuenmayor Toro, 2018). Esto significó el abandono del ingreso por méritos académicos y transformó a la pobreza en una condición de mérito para la admisión universitaria, situación no solo absurda sino monstruosa.

El sistema que desde 2015 hace ingresar a las universidades a aspirantes sin las calificaciones ni conocimientos necesarios es, realmente, un generador de nuevas iniquidades, pues ahora deja fuera a estudiantes con altísimas calificaciones, pero que no pertenecen a los estratos socioeconómicos "D" y "E". Al respecto, Parra-Sandoval y Núñez-Torres (2016, p. 10) señalan que:

El principio de equidad, que supuestamente subyace en esta medida, no llega a cumplirse, en la medida en que los estudiantes de los estratos IV y $\mathrm{V}$ provengan de instituciones oficiales de educación básica y media de baja calidad, donde no están dadas las condiciones para alcanzar niveles 
mínimos de formación, como se ha demostrado e incluso reconocido por el propio gobierno (...)

De hecho, los estudiantes asignados por esta vía en los años 2014, 2015 y 2016, renunciaron a las plazas otorgadas en proporción anual creciente (Luis, E. et al., 2018).

\section{Disminución creciente de la matrícula estudiantil y de los egresados}

Ya a partir de 2009 comienza a verse una afectación negativa matricular en las universidades, la cual no solo es producida por los cambios en la política de selección e ingreso. Es también el resultado del deterioro de las condiciones de vida en el país: el crecimiento de la pobreza general (80\%) y extrema (55\%), las graves dificultades del transporte, la carencia de los servicios públicos, la escasez de dinero en efectivo y el deterioro manifiesto de las actividades institucionales. Las cifras nacionales oficiales de matrícula estudiantil de pregrado señalan un máximo para 2012 que luego decrece $7.2 \%$ en 2013 y $23.3 \%$ en 2014. También se redujo la matrícula de las especializaciones de postgrado en 46\% entre 2009 y 2014 (Parra-Sandoval y Torres-Núñez, 2016).

La Misión Sucre, teóricamente creada para la inclusión universitaria, se vio reducida en 25.9 por ciento en ese mismo lapso, lo que revela el fracaso de la política gubernamental. Este programa demostró desde sus inicios su inefectividad para cumplir su supuesta misión pues, de hecho, generaba una importante deserción estudiantil debida a la existencia de cursantes con muy malas condiciones sociales y económicas a las que se sumaban las limitaciones e incoherencias de los planes, métodos de estudio y funcionamiento de la Misión Sucre (Fuenmayor Toro, et al., 2009).

Para 2017, se denuncia en la Universidad del Zulia reducciones matriculares de $27.3 \%$ en la Escuela de Derecho y $34 \%$ en la Facultad de Agronomía; en el núcleo de la Universidad de Oriente de Puerto Ordaz se reseña una caída de $68.8 \%$ para ese mismo año (Aula Abierta, 2017). En la UCV también se ha reportado una disminución de la matrícula estudiantil entre los años 2012 y 2018 en sus tres facultades más grandes: medicina (-61.9\%), humanidades y educación (-12.1\%) y ciencias económicas y sociales (-36.0\%) (Alfonso, 2018). Los datos que 


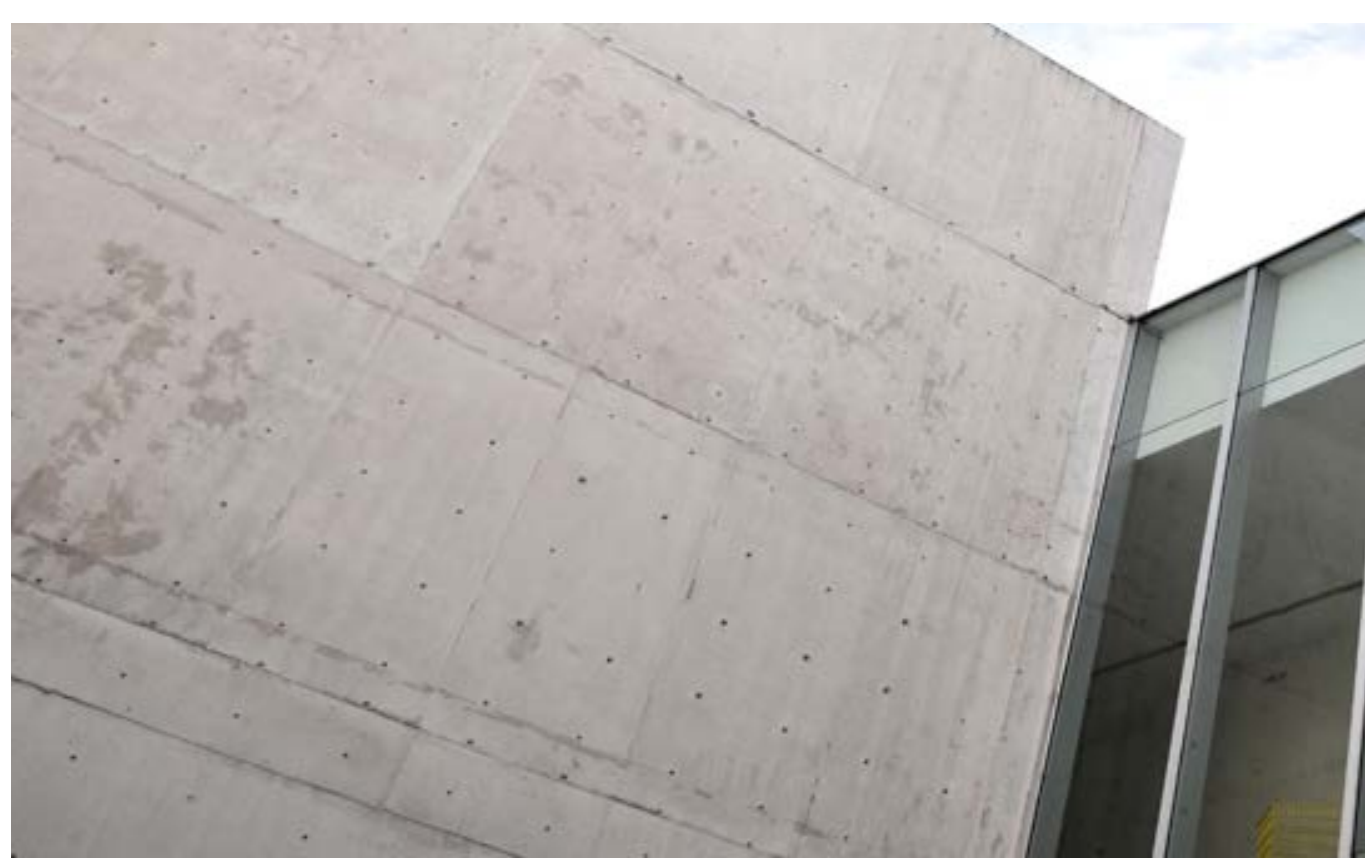

presentamos en la tabla 3a ilustran esta reducción del número de estudiantes a partir de 2013, esta vez en el caso de la Facultad de Ciencias de la UCV que llega a alcanzar más de un 50 \% en 2018.

Como era de esperarse, el número de egresados se ha reducido en los últimos años (Parra-Sandoval y Torres Núñez, 2016). Entre 2010 y 2014, los de pregrado cayeron de 150,107 a 76,911 (-48.8\%), los de las especializaciones bajaron de 9,730 a 5,868 (-39.7\%), los de maestrías de 12,510 a $3,687(-70.5 \%)$ y los de doctorado de 755 a $308(-59.2 \%)$. En la UCV, la reducción de los egresados se produjo no en tan elevadas proporciones (Tabla 3b), lo que puede tener relación con que es una institución situada predominantemente en Caracas, la ciudad menos afectada por la crisis. La tabla $3 b$ muestra que en los primeros seis años del gobierno de Chávez la matrícula se incrementó, reforzando la tesis de García-Guadilla et al. (2006) de que el deterioro es posterior a 2004. Las reducciones habidas junto con la emigración de profesionales generan el grave déficit de estos -principalmente de médicos, ingenieros, docentes y personal técnicoque sufre hoy Venezuela.

La gravedad de la situación es más clara si sabemos que en los niveles previos al universitario hay una importante deserción estudiantil. En un estudio de agosto de 2019, FundaRedes descubre una deserción escolar del $78 \%$ y una asistencia irregular del $66 \%$ de quienes permanecen en el sistema. En las universidades, el estudio apunta también a la presencia de ausentismo y deserción estudiantil, agravada por la inexistencia de servicios de alimentación, salud y transporte, el grave deterioro de las actividades académicas y las renuncias docentes ante los bajísimos sueldos. Por su parte, UNICEF (2019) denuncia que más de un millón de niños están sin escolarizar, lo que niega la propaganda gubernamental de la existencia de escolarización plena en Venezuela. 


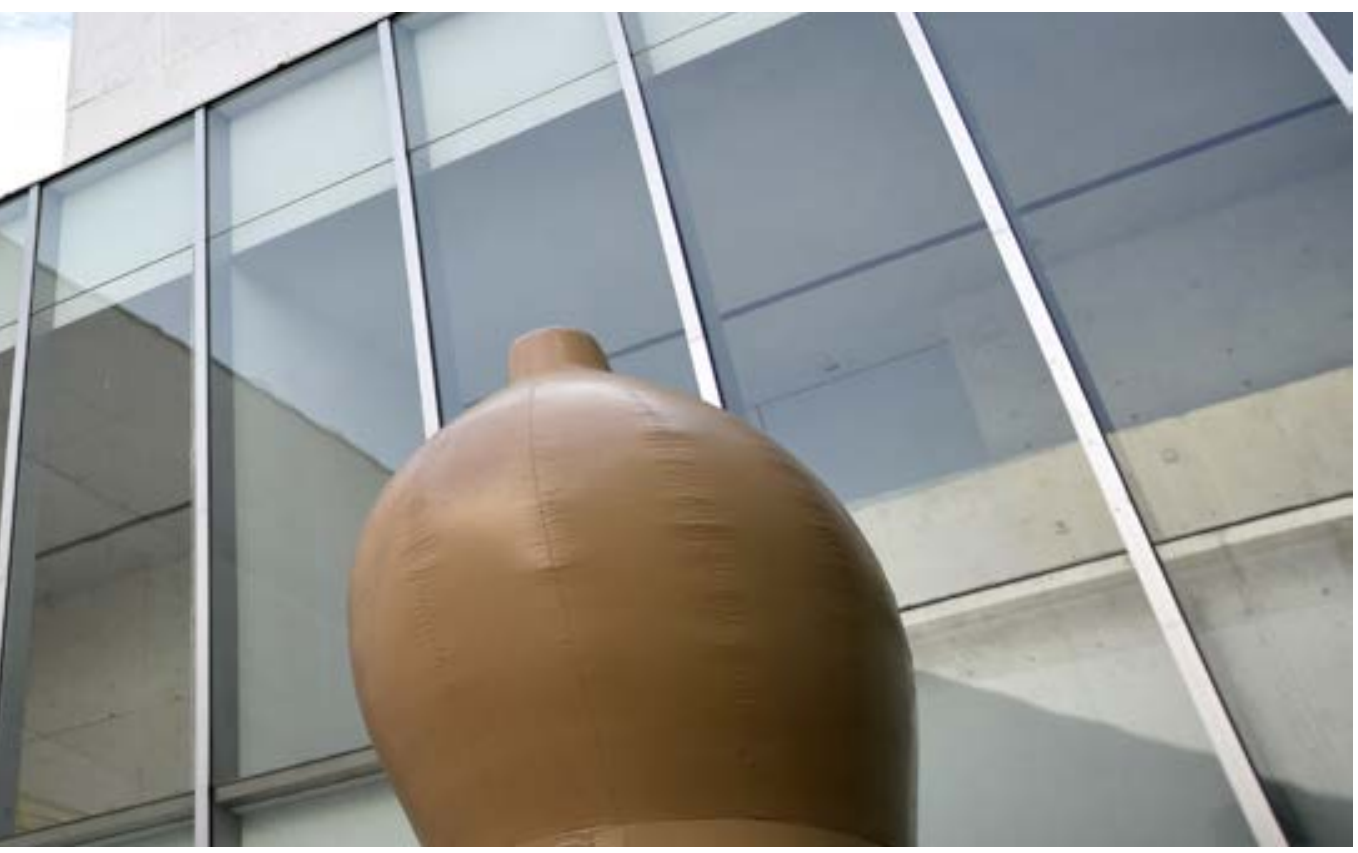

\section{Grave déficit de personal académico}

Este aspecto es uno de los más importantes de la crisis universitaria. La fuga de cerebros académicos amenaza los programas de docencia e investigación, así como a las instituciones, y deja al país en condiciones muy disminuidas de cara al futuro. Ya no solo son las jubilaciones no repuestas de los docentes, ahora se trata de la ida de docentes investigadores en búsqueda de mejores sueldos y condiciones de vida, lo cual los hace marcharse incluso del país. Una demostración palpable de lo señalado es el caso de las renuncias docentes en la Facultad de Ciencias de la UCV (Tabla 4) que se cuadruplicaron entre 2014 y 2018 en relación con las ocurridas entre 2009 y 2013, período de igual duración.

La misma tabla muestra que otro tanto ocurrió, aunque en magnitud menor, con los docentes contratados. En ambos períodos las renuncias docentes fueron mayores en los dos niveles más bajos del escalafón, que acumularon más del $60 \%$ de estas, lo que corresponde al personal académico joven, en formación o recientemente formado (Tabla 4). Las renuncias en los otros sectores siguieron el mismo patrón, con la curiosa excepción del personal administrativo.

Las reposiciones de cargos docentes también se han visto comprometidas, pues los profesionales existentes no atienden las ofertas de las universidades para cubrir las vacantes. Los sueldos no son atractivos, a lo que se suman las pésimas condiciones de trabajo. En la ULA, por ejemplo, durante 2019 en 126 llamados a concursos de oposición no se logró que ningún aspirante participara. Las facultades más afectadas fueron ciencias económicas y sociales, 21 casos; medicina, 20; ingeniería, 14; odontología, 12; ciencias, 9, y humanidades y educación, 7. El núcleo universitario más afectado fue el del estado Táchira con 22 casos. 
La tabla 5 muestra la evolución de los sueldos de los docentes investigadores venezolanos desde los años setenta. Se observa cómo se incrementaron hasta 1982, cuando alcanzaron niveles aceptables internacionalmente. La crisis económica de entonces más la caída de los precios petroleros indujeron una reducción salarial muy drástica, que alcanzó más del $80 \%$ y que se prolongó hasta finales de los ochenta. Se inicia luego una muy modesta recuperación que se potencia a comienzos de este siglo en el gobierno de Hugo Chávez, cuando alcanza un $72 \%$ de los niveles salariales de 1982, dato que ratifica la tesis que el deterioro se desarrolló luego de los primeros seis años de gobierno de Chávez. La tabla 5 nos muestra la reducción posterior de los sueldos que se hace brutal en 2015 y sigue hasta hoy cuando se desploma a montos muy por debajo del nivel de subsistencia.

\section{Deterioro de la investigación científica}

Otra área, cuyo quebranto ilustra la crisis del sector universitario, es la relativa a la creación de conocimientos. Es imposible pensar que las reducciones en el número de los profesores investigadores, la merma en las maestrías y doctorados y las limitaciones de los servicios (agua potable, suministro eléctrico y telecomunicaciones) iban a dejar incólume la producción de conocimientos de las universidades, la actividad académica más sensible a estas variaciones. De hecho, en la UCV los programas financiados por el Consejo de Desarrollo Científico y Humanístico (CDCH), como el de formación de investigadores (becas) y los de proyectos de investigación y de asistencia a eventos científicos, se han ido reduciendo hasta casi desaparecer (Tabla 6).

La fuga de talentos afecta seriamente a las ciencias y la tecnología del país, pues lo deja sin una masa crítica de científicos, que imposibilita tener la generación de relevo necesaria y que será muy difícil corregir. De toda la emigración de científicos venezolana desde 1960 hasta 2014 (1,783 investigadores), el 84.8\% se produjo desde 1999 (Requena y Caputo, 2016), afectando mayormente a los investigadores responsables de más del 30\% de las publicaciones hechas en el país. Y esto ocurría hasta hace 5 años, cuando aún estábamos lejos del grado actual de deterioro.

Esta investigación revela datos que soportan algunos de nuestros señalamientos. "Durante los primeros años del siglo XXI hasta el año 2008, la tasa anual de incremento en el número de publicadores se mantuvo estable e igual a la última tasa del siglo anterior, del orden de 80 publicadores por año" (Requena y Caputo, 2016, p. 447), lo que significa que el deterioro arrancó con posterioridad. Los científicos emigrantes son principalmente personas entre 30 y 45 años, lo que se corresponde con la tendencia ya señalada de las renuncias docentes ocurridas en la Facultad de Ciencias de la UCV (Tabla 4).

\section{Las limitaciones presupuestarias}

Las universidades venezolanas se han quejado siempre de ser maltratadas financieramente por el Estado. De hecho, en 1868 el claustro de la UCV refiere los sufrimientos de la Real y Pontificia Universidad de Caracas que 
permaneció "estrecha, pobre y arrimada al Seminario de Santa Rosa hasta el año 1827 en que regresó a esta capital el Libertador Presidente Simón Bolívar" (Leal, 1981, p. 30). En ese momento arrancan tres décadas de holgura financiera y crecimiento institucional gracias a las medidas tomadas por el Libertador, período que finaliza con la abolición de la esclavitud que deja a las haciendas entregadas por Bolívar sin mano de obra y la guerra federal y el despojo que le hace el presidente Guzmán Blanco de sus bienes inmuebles.

A partir de ese momento se reinicia un largo período de limitaciones financieras, acompañadas incluso de cierres institucionales hasta 1958, cuando luego de la caída del gobierno de Pérez Jiménez, un segundo período de holgura financiera se presenta con el advenimiento del régimen democrático representativo. Se genera un crecimiento y desarrollo de todo el sistema universitario nacional que se mantiene hasta principio de los años ochenta, cuando se desata la crisis económica de esa época (Fuenmayor Toro, 2002).

Lo señalado se muestra en la tabla 7 , que presenta el comportamiento del presupuesto de las universidades desde 1964 hasta el presente. En la misma, se tuvo cuidado en presentar siempre las cifras mínimas y máximas de las fluctuaciones ocurridas. Se observa el constante incremento presupuestario desde 1964 hasta alcanzar el monto de 1981, hacia la primera mitad del período democrático representativo. Luego ocurre una importante caída que se mantiene hasta finales de los ochenta, debida a la crisis económica de entonces que causó severos daños a las instituciones en su infraestructura, equipamiento, bibliotecas, personal académico, bienestar social y sueldos docentes y de los trabajadores en general.

La recuperación financiera casi total hacia el final de la década de los noventa es seguida, en los primeros años de este siglo, por una más que duplicación del presupuesto universitario que para 2001 alcanza la mayor cifra en dólares en términos absolutos que haya sido otorgada a las universidades (Tabla 7). Los montos se reducen luego como consecuencia de los hechos políticos ocurridos entonces: golpe de Estado en 2002, huelga general empresarial (lock out) en 2002 y el sabotaje a la industria petrolera a finales de 2002 e inicios de 2003. En 2008 se produjo una recuperación de un $85 \%$ del presupuesto de 2001 para luego iniciar un descenso con motivo del comienzo de la crisis económica actual que llevó los montos presupuestarios a las ínfimas cifras de 2015 y 2018.

\section{El deterioro más allá de las cifras}

Las universidades venezolanas son hoy un espectro de lo que alguna vez fueron. Las cifras no nos hacen imaginar la situación dantesca por la que atraviesan. A los infames presupuestos que no permiten funcionar, ni mucho menos mantener lo existente, se une el absoluto abandono por parte del Estado en lo que respecta a la seguridad de personas y bienes. Además, todas las universidades han sido judicialmente impedidas de renovar a sus autoridades como lo ordenan la Constitución y las leyes, lo que lleva a tener las mismas autoridades centrales y decanales desde hace más de diez años, con el normal agotamiento que esto significa. 
Se quiere imponer un sistema electoral que trata a estas instituciones como si fueran pequeñas repúblicas. No se quiere entender que las instituciones basadas en el conocimiento son de carácter meritocrático, por lo que la democracia republicana no les es aplicable, como no lo es a las fuerzas armadas de ningún país, ni a los hospitales, ni a la iglesia. Comprender que no se puede elegir por votaciones al piloto de un avión, ni al ingeniero civil que construirá una autopista, ni al cirujano que actuará en un quirófano, parece estar fuera de las posibilidades intelectuales de quienes hoy gobiernan. No se elige doctores ni profesores de ningún grado en asambleas, como tampoco los graduados son los estudiantes más votados por sus compañeros, ya que en todas estas actividades el conocimiento es el que determina las posiciones a ocupar.

Menos se entiende que estudiantes y profesores son iguales entre sí en sus derechos civiles y políticos, pero no en sus jerarquías académicas. Un estudiante recién iniciado no es académicamente igual a su compañero a punto de graduarse, pues si lo fuera para qué pasar por cinco o seis años de estudios, entrenamientos y formación. Las relaciones entre un tutor de doctorado y el estudiante graduado no es una relación de poder. Aquél no es el jefe de este, su autoridad deriva del conocimiento que tiene, del cual el estudiante se beneficiará y por ello incluso es él quien lo escoge.

Las universidades han sido desmanteladas por el hampa en actos vandálicos de naturaleza política, ocupación ilegal de sus espacios, impedimento físico de sus labores, desvalijamiento de sus dependencias, sustracción de material especializado de sus instalaciones, atracos colectivos en aulas de clase y hasta asesinatos. La Universidad de Oriente es una de las más afectadas, como lo describe Fariñas (2019) dramáticamente.

Todas las instituciones universitarias y educativas, en general, son víctimas de esta gravísima situación que queda impune ante la indolencia gubernamental. La Universidad del Zulia tiene paralizada muchas de sus actividades. La UCV es víctima permanente, en especial su Instituto de Medicina Tropical, Facultad de Medicina, al que un grupo parapolicial de delincuentes le declaró la guerra y lo ha desvalijado una treintena de veces en dos años. En algunas de las incursiones se han llevado hasta animales de laboratorio inoculados con bacterias y parásitos (Freisler, 2019).

\section{Conclusiones}

Podemos concluir objetivamente que la universidad venezolana atraviesa uno de los peores momentos de sus casi 300 años de historia, y ciertamente el peor de los últimos 60 años. Su actual orfandad se debe a las políticas de hostigamiento de todo tipo y cerco financiero impuestos por el gobierno nacional, iniciados a partir de 2005 y profundizados con posterioridad a 2012. Ha sido muy grave que internamente haya docentes, que se hayan prestado cobardemente a patrocinar las actitudes infames contra la institución. También que no haya habido la suficiente consecuencia por preservar la academia y defender la institución por parte de sus autoridades, muchas veces ocupadas más de sus proyectos políticos nacionales o incluso, en forma aberrante, de sus negocios ilícitos. 
Rescatar la universidad va a ser un trabajo muy difícil y de largo aliento. Dos a tres décadas, si se asume como prioridad nacional y se ejecuta con ahínco y constancia. Recuperar la planta de docentes-investigadores bien formados, la infraestructura física, las dotaciones de todo tipo y la mística del trabajo académico creativo y de calidad son los retos fundamentales. Erradicar el facilismo y el igualitarismo ideologizado serían las premisas principales.

\section{Anexo}

Tabla 1. Publicaciones periódicas oficiales del CNU en distintos momentos

\begin{tabular}{|c|c|c|}
\hline 1998 & 2004 & 2019 \\
\hline $\begin{array}{l}\text { Libro Oportunidades de } \\
\text { Estudio. Publicación anual } \\
\text { en formato físico }\end{array}$ & $\begin{array}{c}\text { Libro Oportunidades } \\
\text { de Estudio. Publicación } \\
\text { anual en formatos físico y } \\
\text { electrónico }\end{array}$ & $\begin{array}{c}\text { Libro Oportunidades } \\
\text { de Estudio. Publicación } \\
\text { electrónica }\end{array}$ \\
\hline $\begin{array}{l}\text { Separata del Boletín } \\
\text { Estadístico de Educación } \\
\text { Superior (Información } \\
\text { parcial del período 1989- } \\
\text { 1994*). Publicación en } \\
\text { formato físico }\end{array}$ & $\begin{array}{l}\text { Boletín Estadístico de } \\
\text { Educación Superior } \\
\text { (Información completa } \\
\text { del periodo 1995-2002). } \\
\text { Publicación anual en } \\
\text { formato físico }\end{array}$ & \\
\hline & $\begin{array}{c}\text { El Proceso Nacional } \\
\text { de Admisión en cifras. } \\
\text { Publicación anual en físico }\end{array}$ & ---- \\
\hline $\begin{array}{l}\text { Resoluciones del CNU. } \\
\text { Publicación discontinua } \\
\text { (Información de 1988) }\end{array}$ & $\begin{array}{l}\text { Resoluciones del CNU. } \\
\text { Publicación anual en físico } \\
\text { (Información del período } \\
\text { 1989-2003) }\end{array}$ & \\
\hline & $\begin{array}{c}\text { Publicación periódica } \\
\text { monográfica Cuadernos } \\
\text { OPSU }\end{array}$ & ---- \\
\hline & $\begin{array}{l}\text { Publicación periódica } \\
\text { divulgativa Opciones }\end{array}$ & \\
\hline & $\begin{array}{c}\text { Publicación semestral de } \\
\text { investigación Experiencia } \\
\text { Universitaria }\end{array}$ & ---- \\
\hline
\end{tabular}

Nota. * El último Boletín Estadístico se publicó en 1994 con la información de 1988. En 2004, OPSU publica una nueva Separata para completar la información faltante. 


\section{Tabla 2. Construcciones en la Universidad de Carabobo (2000-2008) con financiamiento oficial nacional}

Facultad de Ciencia y Tecnología: edificios de química, computación, auditorio, aulas, cafetín; equipamiento; edificios decanato, biblioteca, aulas y laboratorios (biología, matemática, física y humanidades); obras de arte; estacionamientos; paisajismo.

Facultad de Ciencias de la Educación: edificio de facultad: aulas, laboratorios, auditorio, servicios, cafetín; estacionamientos; paisajismo.

Facultad de Ciencias Jurídicas y Políticas: dos edificios, estacionamientos, obras de arte y paisajismo.

Facultad de Ciencias de la Salud: edificio de biomédica, decanato, aulas, postgrado y ambulatorio; equipamiento; remodelación de siete pabellones; paisajismo; obras de arte; edificios enfermería, bioanálisis y biblioteca para ciencias de la salud, odontología y biología; escuela de medicina de Puerto Cabello; estacionamientos.

Facultad de Odontología: construcción del 90\% del edificio de aulas; no se culminó en 2009. Reconstrucción de consultorios, equipamiento consultorios y laboratorios.

Facultad de Ciencias Económicas y Sociales: construcción edificio de postgrado, módulo de servicios y cancha de básquetbol. Restauración y remodelación de departamentos, cátedras y sanitarios. Obras de arte.

Facultad de Ingeniería: remodelación de auditorios, departamentos, cátedras, sanitarios y cancha de básquetbol. Construcción cafetín y comedor.

Instalaciones deportivas: construcción palacio de combate, seis canchas de tenis, canchas de fútbol, pista de atletismo, gimnasios cubiertos, piscina olímpica y de salto.

Núcleo La Morita: edificio de aulas de FACES, aulas de ciencias de la salud, cafetín, comedor estudiantil, gimnasio cubierto.

Construcción Instituto Experimental Simón Bolívar, Consejo Universitario y Centro de Estimulación Integral, comedor y planta física (Bárbula); Casa de la Luz (Naguanagua) equipada (atención médico odontológica, laboratorio, Rayos X y jurídica de la población).

Construcción edificio de Patrimonio Histórico (centro de Valencia), recuperación Anfiteatro de Bárbula, cerrado por diez años; remodelación Galería Braulio Salazar, construcción auditorio casa de las Brisas (Escuela Sistema de Orquestas Simón Bolívar).

Aula Magna: infraestructura y cerramiento: 2,631 espectadores. Funcionó entre 2013 y 2018.

Nota. Datos obtenidos de la Universidad de Carabobo. Elaboración propia. No hay nuevas construcciones desde 2009 .

46 Universidades $\mid$ núm. 83, enero-marzo $2020 \mid$ UDUAL $\mid$ DOI:https://doi.org/10.36888/udual.universidades.2020.83.75 Luis Fuenmayor Toro $\mid$ Deterioro de la universidad venezolana en los últimos 15 años 
Tabla 3a. Disminución absoluta y relativa de la matrícula estudiantil de la Facultad de Ciencias UCV en los últimos años

\begin{tabular}{|c|c|c|}
\hline Años & Número & Variación respecto a 2003 \\
\hline 2013 & 3,038 & ------ \\
\hline 2014 & 2,981 & $-1.9 \%$ \\
\hline 2015 & 2,708 & $-10.9 \%$ \\
\hline 2016 & 2,355 & $-22.5 \%$ \\
\hline 2017 & 2,196 & $-27.7 \%$ \\
\hline 2018 & 1,431 & $-52.9 \%$ \\
\hline
\end{tabular}

Nota. Datos de la Oficina de Control de Estudios. Cálculos propios.

Tabla 3b. Variación del número de egresados de la UCV en los años señalados

\begin{tabular}{|c|c|c|}
\hline Años & Número & Variación respecto a 2004 \\
\hline 1994 & 3,920 & -27.5 \\
\hline 1999 & 4,960 & $-8.2 \%$ \\
\hline 2004 & 5,404 & ------ \\
\hline 2009 & 4,778 & $-11.6 \%$ \\
\hline 2014 & 4,124 & $-23.7 \%$ \\
\hline 2018 & 3,701 & $-31.5 \%$ \\
\hline
\end{tabular}

Nota. Datos de la Secretaría de la UCV. Cálculos propios.

Tabla 4. Renuncias de personal en los lapsos señalados, expresadas como porcentaje del total de renuncias en ambos lapsos por tipo de personal y renuncias docentes por categoría académica como porcentaje del total de renuncias de los docentes de planta. Facultad de Ciencias, UCV

\begin{tabular}{|c|c|c|}
\hline Tipo de personal & 2009-2013 & 2014-2018 \\
\hline Docentes de planta & $21.6 \%$ & $78.4 \%$ \\
\hline Docentes contratados & $39.6 \%$ & $60.4 \%$ \\
\hline Instructores & \multicolumn{2}{|c|}{$30.0 \%$} \\
\hline Asistentes & \multicolumn{2}{|c|}{$32.9 \%$} \\
\hline Agregados & \multicolumn{2}{|c|}{$24.3 \%$} \\
\hline Asociados & \multicolumn{2}{|c|}{$7.1 \%$} \\
\hline Titulares & \multicolumn{2}{|c|}{$5.7 \%$} \\
\hline Técnico y de servicio & $22.0 \%$ & $17.1 \%$ \\
\hline Administrativo & $26.8 \%$ & $34.1 \%$ \\
\hline Obrero & $15.4 \%$ & $84.6 \%$ \\
\hline
\end{tabular}

Nota. Datos de la Dirección de Recursos Humanos. Cálculos propios. 
Tabla 5. Variación del sueldo básico mensual en dólares (US) del personal académico universitario venezolano a máxima dedicación en los niveles señalados

\begin{tabular}{|c|c|c|}
\hline Años & Instructor & Profesor Titular \\
\hline $1974^{*}$ & 837 & 1,884 \\
\hline $1982^{* *}$ & 1,671 & 3,472 \\
\hline $1983^{* *}$ & 801 & 1,664 \\
\hline $1986^{* *}$ & 509 & 1,057 \\
\hline $1988^{* *}$ & 321 & 667 \\
\hline $1992^{* *}$ & 522 & 1,121 \\
\hline $1998^{* *}$ & 534 & 1,144 \\
\hline $2001^{* *}$ & 1,088 & 2,573 \\
\hline $2003^{* *}$ & 589 & 1,392 \\
\hline $2010^{* * *}$ & 426 & 924 \\
\hline $2015^{* * *}$ & 39 & 56 \\
\hline 2019 abril\# & 6.19 & 8.75 \\
\hline 2019 septiembre\#\# & 3.62 & 5.00 \\
\hline
\end{tabular}

Nota. Datos de * Peralta (2002); ** UCV (2006); *** Cálculos propios; \# García (2019); \#\# Muñoz (2019).

Tabla 6. Número de programas académicos financiados por el CDCH de la UCV en las últimas décadas

\begin{tabular}{|c|c|c|c|}
\hline Años & $\begin{array}{c}\text { Becarios en el } \\
\text { exterior }\end{array}$ & $\begin{array}{c}\text { Proyectos de } \\
\text { investigación }\end{array}$ & $\begin{array}{c}\text { Asistencia a } \\
\text { eventos científicos }\end{array}$ \\
\hline 1980 & 174 & 73 & ----- \\
\hline 1990 & 64 & 179 & 530 \\
\hline 2000 & 148 & 230 & 1,389 \\
\hline 2010 & 39 & 164 & 33 \\
\hline 2017 & 14 & 8 & 5 \\
\hline
\end{tabular}

Nota. Datos proporcionados por el CDCH de la UCV. 
Tabla 7. Presupuestos del sector universitario venezolano en millones de dólares (US) en los años señalados

\begin{tabular}{|c|c|}
\hline Años & Dólares \\
\hline $1964^{*}$ & 43 \\
\hline $1975^{* *}$ & 370 \\
\hline $1978^{* *}$ & 603 \\
\hline $1981^{* *}$ & 1,050 \\
\hline $1984^{* *}$ & 345 \\
\hline $1988^{* *}$ & 274 \\
\hline $1991^{* *}$ & 542 \\
\hline $1994^{* *}$ & 916 \\
\hline $1996^{* *}$ & 522 \\
\hline $1998^{* *}$ & 989 \\
\hline $2000^{* *}$ & 1,630 \\
\hline $2001^{* *}$ & 2,172 \\
\hline $2003^{* *}$ & 1,330 \\
\hline $2005^{* *}$ & 1,666 \\
\hline $2008 \#$ & 1,852 \\
\hline $2012 \#$ & 1,300 \\
\hline $2015 \#$ & 85 \\
\hline $2018 \#$ & 39 \\
\hline
\end{tabular}

Nota. Cálculos de * UCV (1992); ** UCV (2006); \# Cálculos propios con cifras de las leyes anuales de presupuesto y su transformación en dólares con el valor del dólar paralelo (libre) calculado anualmente como el promedio de sus valores mensuales. 


\section{Referencias}

Alfonso, G. (2018). El aumento de la matrícula universitaria y la mentira como política de Estado en Venezuela. El Libertario. Diciembre. Extraído el 8 de septiembre de 2019 desde http://periodicoellibertario.blogspot.com/2018/12/ el-aumento-de-la-matricula.html .

Aula Abierta (2017). Informe preliminar: Deserción estudiantil en Venezuela. Extraído el 8 de septiembre de 2019 desde http://aulaabiertavenezuela.org/wp-content/uploads/2017/12/AULA-ABIERTA-VENEZUELA-INFORME-PRELIMINAR-DESERCI\%C3\%93N-UNIVERSITARIA-EN-VENEZUELA.pdf.

Fariñas, Á. R. (2019). Saqueo generalizado a la escuela de ciencias de la Universidad de Oriente. La academia agoniza. Aporrea.org 13 de febrero. Extraído el 15 de septiembre de 2919 desde https://t.co/NLe5J2kY4L?amp=1.

Fondo de las Naciones Unidad para la infancia (UNICEF) (2019). Más de un millón de niños en Venezuela está sin escolarizar. Voz de América, septiembre. Extraído el 20 de septiembre de 2019 desde https:/www.voanoticias.com/a/ unicef-mas-de-un-millon-de-menores-en-venezuela-estan-sin-escolarizar-/5091669.html.

Freisler, E. (2019). Médicos venezolanos temen brotes epidémicos letales. El Nuevo Herald 29 de mayo. Extraído el 28 de octubre de 2019 desde https:/www. elnuevoherald.com/noticias/mundo/america-latina/venezuela-es/article230931753.html.

Fuenmayor Toro, L. (2002). Historia, desarrollo y perspectivas del sector universitario venezolano. Cuadernos OPSU. $N^{\circ} 5$, págs. 1-36, Caracas, Venezuela. http:// saber.ucv.ve/handle/123456789/17298.

Fuenmayor Toro, L. (2004). Inaugurada primera aldea universitaria bolivariana. Sol de Margarita. Pág. 10, 12 de octubre, Porlamar, Venezuela.

Fuenmayor Toro, L. y Mejías, E. (2004). Criterios de admisión de los aspirantes a la educación superior y su influencia en la conformación de los mejores. Experiencia Universitaria. Vol. 1, № 2, págs. 9-36, Caracas, Venezuela. http:// saber.ucv.ve/handle/123456789/17423.

Fuenmayor Toro, L. (2009). La calidad de la universidad venezolana. Cuadernos OPSU. $\mathrm{N}^{\circ} 11$, págs. 3-21, Caracas, Venezuela. http://saber.ucv.ve/handle/123456789/17208.

Fuenmayor Toro, L., Marín, M., Silvera, F. y Jaimes, J. (2009). Deserción estudiantil en la Misión Sucre: Primer Programa de Iniciación Universitaria en la Parroquia Macarao de Caracas. Educere. Vol. 13, № 44, págs. 751-769, Mérida, Venezuela.

FUNDAREDES. (2019). Informe técnico sobre consulta educativa en Venezuela. Agosto. Extraído el 29 de octubre de 2019 desde https://www.fundaredes.org/wp-content/uploads/2019/09/DOC-20190901-WA0074.pdf.

García-Guadilla C., Montilva, L., Acedo, M., Lepage, B. y Otero, S. (2006). Proyecto CINDA. Educación Superior en Iberoamérica 2006. Informe de Venezuela. Extraído el 19 de agosto de 2019 desde http://www.carmengarciaguadilla.com/ articulos/2006_EducSup_en_Iberoamerica.pdf. 
García, I. (2019). Consejo Universitario debate tablas salariales impuestas al personal universitario. UCV Noticias. Extraído el 14 de octubre de 2019 desde https://ucvnoticias.wordpress.com/2019/04/25/consejo-universitario-debate-las-tablas-salariales-impuestas-al-personal-universitario/.

Leal, I. (1981). Historia de la UCV. Ediciones del Rectorado de la UCV, Caracas, Venezuela: Imprenta Universitaria.

Luis, E., Cortázar, J. M. y Fuenmayor Toro, L. (2018). La admisión universitaria en Venezuela hasta 2007. Comparación del proceso nacional CNU-OPSU con los mecanismos universitarios de ingreso, en especial el de la UCV. Revista Venezolana de Análisis de Coyuntura. Vol. 24, № 1, págs. 155-178, Caracas, Venezuela. http://saber.ucv.ve/ojs/index.php/rev_ac/article/view/15580.

Muñoz, Á. (2019). El sueldo más alto de un profesor universitario es de 5,90 \$ mensuales. 2 de septiembre. Extraído el 14 de octubre de 2019 desde https://twitter. com/AlvaroMunoz/status/1168649866408529920.

OPSU (2001). Proyecto "Alma Mater" para el mejoramiento de la calidad y la equidad de la educación universitaria en Venezuela. Cuadernos OPSU. No 1,43 P, Caracas, Venezuela.

OPSU. (2004). Presentación. Proyecto Alma Mater “Gran Sabana”, La Aldea Universitaria Bolivariana. Cuadernos OPSU. № 9, págs. v-vii, Caracas, Venezuela.

Parra-Sandoval, M. C. y Torres-Núñez, L. E. (2016). Educación Superior en Iberoamérica. Informe 2016. Informe Nacional: Venezuela. Extraído el 15 de agosto de 2019 desde https://cinda.cl/publicacion_archivos/educacion-superior-en-iberoamerica-informe-2016-informe-nacional-venezuela/.

Peralta, M. (2002). Evolución de los sueldos universitarios. Aporrea.org, 24 de noviembre. Extraído el 14 de octubre de 2019 desde https://www.aporrea.org/ actualidad/a1083.html.

Requena, J. y Caputo, C. (2016). Pérdida de talento en Venezuela: migración de investigadores. Interciencia. Vol. 41, $\mathrm{N}^{\circ} 7$, págs. 444-453, Caracas, Venezuela.

Sarco-Lira, A. y Fuenmayor Toro, L. (2004). Evaluación de los nuevos procedimientos utilizados en la fase de asignación del Proceso Nacional de Admisión. Experiencia Universitaria. Vol. 2, № 3, págs. 35-69, Caracas, Venezuela.

Universidad Central de Venezuela. (1992). 30 Años del Presupuesto de la UCV. 1960-1990. Vicerrectorado Administrativo, Caracas, Venezuela: Imprenta Universitaria.

Universidad Central de Venezuela. (2006). 30 Años del Presupuesto de la UCV.19752004. Vicerrectorado Administrativo, Caracas, Venezuela: Puligráficas 66. 